\title{
Solutions to Problems
}

Problem 1 The Galilean transformation for infinitesimals is $d x=d x^{\prime}+v d t^{\prime}, d y=$ $d y^{\prime}, d z=d z^{\prime}$ and $d t=d t^{\prime}$. Substituting this into equation (1.7) $d s^{2}=c^{2} d t^{2}-d x^{2}-$ $d y^{2}-d z^{2}$ gives $d s^{2}=c^{2} d t^{\prime 2}-d x^{\prime 2}-d y^{\prime 2}-d z^{\prime 2}-2 v d x^{\prime} d t^{\prime}-v^{2} d t^{\prime 2} \neq d s^{\prime 2}$. A similar result follows for the sum of squares. In general the reason why we cannot find an invariant under the Galilean transformation is that the time component is fixed, so changes in the $x$ component cannot cancel changes in the time component.

Problem 2 Multiplying $v^{\prime \mu}=\frac{\partial x^{\prime \mu}}{\partial x^{\nu}} v^{\nu}$ by $\frac{\partial x^{\rho}}{\partial x^{\prime \mu}}$ gives $\frac{\partial x^{\rho}}{\partial x^{\prime \mu}} v^{\prime \mu}=\frac{\partial x^{\rho}}{\partial x^{\prime \mu}} \frac{\partial x^{\prime \mu}}{\partial x^{\nu}} v^{\nu}=\delta_{\nu}^{\rho} v^{\nu}=v^{\rho}$. Change the free index $\rho$ to $\mu$ and the dummy index $\mu$ to $\nu$ and the result follows. A similar procedure yields the inverse transformation of the covariant components.

Problem 3 The scalar product $v^{\mu} v_{\mu}=\frac{\partial x^{\mu}}{\partial x^{\prime \nu}} v^{\prime \nu} \frac{\partial x^{\prime}}{\partial x^{\mu}} v_{\rho}^{\prime}=\delta_{\nu}^{\rho} v^{\prime \nu} v_{\rho}^{\prime}=v^{\prime \nu} v_{\nu}^{\prime}$. Hence the scalar product is an invariant.

\section{Problem 4}

$$
\nabla_{\nu}\left(A^{\mu} B_{\mu}\right)=\partial_{\nu}\left(A^{\mu} B_{\mu}\right)
$$

Differentiating gives

$$
B_{\mu} \nabla_{\nu} A^{\mu}+A_{\mu} \nabla_{\nu} B^{\mu}=B_{\mu} \frac{\partial A^{\mu}}{\partial x^{\nu}}+A^{\mu} \frac{\partial B_{\mu}}{\partial x^{\nu}} .
$$

Now

$$
\nabla_{\nu} A^{\mu}=\frac{\partial A^{\mu}}{\partial x^{\nu}}+\Gamma_{\nu \rho}^{\mu} A^{\rho}
$$

So

$$
A^{\mu} \nabla_{\nu} B_{\mu}=A^{\mu} \frac{\partial B_{\mu}}{\partial x^{\nu}}-B_{\mu} \Gamma_{\nu \rho}^{\mu} A^{\rho}
$$

Interchange the indices $\mu$ and $\rho$ in the last term and cancelling the $A^{\mu}$ gives

$$
\nabla_{\nu} B_{\mu}=\frac{\partial B_{\mu}}{\partial x^{\nu}}-\Gamma_{\nu \mu}^{\rho} B_{\rho}
$$




\section{Problem 5 Let}

$$
\begin{aligned}
L_{V} A^{\mu} & =V^{\nu} \nabla_{\nu} A^{\mu}-A^{\nu} \nabla_{\nu} V^{\mu} \\
& =V^{\nu}\left[\frac{\partial A^{\mu}}{\partial x^{\nu}}+\Gamma_{\nu \rho}^{\mu} A^{\rho}\right]-A^{\nu}\left[\frac{\partial V^{\mu}}{\partial x^{\nu}}+\Gamma_{\nu \rho}^{\mu} V^{\rho}\right] \\
& =V^{\nu} \partial_{\nu} A^{\mu}-A^{\nu} \partial_{\nu} V^{\mu}+\Gamma_{\nu \rho}^{\mu} A^{\rho} V^{\nu}-\Gamma_{\nu \rho}^{\mu} A^{\nu} V^{\rho} .
\end{aligned}
$$

The last two terms cancel, as the dummy indices can be relabelled to make them identical, so it is legitimate to replace the partial derivatives by covariant derivatives in the Lie derivative.

Problem 6 The tensor transformation law for a second rank contravariant tensor is

$$
T^{\mu \nu}=\frac{\partial x^{\mu}}{\partial x^{\prime \sigma}} \frac{\partial x^{\nu}}{\partial x^{\prime \rho}} T^{\prime \sigma \rho} .
$$

The co-variant derivative is

$$
\nabla_{\sigma} T^{\mu \nu}=\frac{\partial T^{\mu \nu}}{\partial x^{\sigma}}+\Gamma_{\sigma \rho}^{\mu} T^{\rho \nu}+\Gamma_{\sigma \lambda}^{\nu} T^{\rho \lambda} .
$$

The Lie derivative is

$$
£_{V} T^{\mu \nu}=V^{\sigma} \nabla_{\sigma} T^{\mu \nu}-T^{\sigma \nu} \nabla_{\sigma} V^{\mu}-T^{\mu \sigma} \nabla_{\sigma} V^{\nu} .
$$

Problem 7 We have $g^{\alpha \beta} g_{\alpha \beta}=4$. Therefore

$$
g^{\alpha \beta}\left(R_{\alpha \beta}-\frac{1}{2} g_{\alpha \beta} R\right)=R-2 R=-R=g^{\alpha \beta} T_{\alpha \beta}=0 .
$$

Problem 8 We have from problem 6

$$
\begin{aligned}
£_{K} g^{\mu \nu} & =k^{\alpha} \nabla_{\alpha} g^{\mu \nu}-g^{\alpha \nu} \nabla_{\alpha} k^{\mu}-g^{\mu \alpha} \nabla_{\alpha} k^{\nu} \\
& =0-\left(\nabla^{\nu} k^{\mu}+\nabla^{\mu} k^{\nu}\right),
\end{aligned}
$$

from which the result follows.

Problem 9 From the metric the proper circumference of the curve $r=$ constant in the plane $\theta=\pi / 2$ at constant time $t$ is $2 \pi r$. At the event horizon $r=2 m$. So the proper circumference of a Schwarzschild black hole is $4 \pi m$. From the metric the area of the surface $r=$ constant, $t=$ constant is

$$
\text { Area }=r^{2} \int_{0}^{2 \pi} d \phi \int_{-\pi / 2}^{\pi / 2} \sin \theta d \theta
$$

The angular integral is the area of the unit sphere i.e. $4 \pi$ and $r=2 m$ so the black hole area is

$$
A_{h}=16 \pi m^{2} .
$$


Problem 10 Expanding the scalar product $g^{\mu \nu} u_{\mu} u_{\nu}=1$ gives

$$
g^{00}\left(u_{0}\right)^{2}+g^{11}\left(u_{1}\right)^{2}+g^{33}\left(u_{3}\right)^{2}=1 .
$$

Inserting the values of the metric coefficients, putting $u_{0}=E$ and $u_{3}=-L$ and rearranging gives

$$
\left(1-\frac{2 m}{r}\right)\left(u_{1}\right)^{2}=\frac{E^{2}}{\left(1-\frac{2 m}{r}\right)}-\frac{L^{2}}{r^{2}}-1 .
$$

Now $u_{1}=g_{11} u^{1}=-(1-2 m / r)^{-1} d r / d \tau$ so finally we get

$$
\left(\frac{d r}{d \tau}\right)^{2}=E^{2}-\left(1+\frac{L^{2}}{r^{2}}\right)\left(1-\frac{2 m}{r}\right) .
$$

Problem 11 The geodesic equations are

$$
\frac{d u^{\mu}}{d \tau}+\Gamma_{\sigma \rho}^{\mu} u^{\sigma} u^{\rho}=0 .
$$

The $\mu=1$ equation, in physical units, is

$$
\frac{d^{2} r}{d \tau^{2}}+\Gamma_{00}^{1} c^{2}\left(\frac{d t}{d \tau}\right)^{2}+\Gamma_{22}^{1}\left(\frac{d \theta}{d \tau}\right)^{2}+\Gamma_{33}^{1} c^{2}\left(\frac{d \phi}{d \tau}\right)^{2}=0
$$

With the Newtonian metric, $\Gamma_{00}^{1}=m / r^{2}, \Gamma_{22}^{1}=-r$ and $\Gamma_{33}^{1}=-r \sin ^{2} \theta$ and at low speeds, $d t \sim d \tau$. With these values the $\mu=1$ equation becomes

$$
\frac{d^{2} r}{d t^{2}}=\frac{G M}{r^{2}}+r\left(\frac{d \phi}{d t}\right)^{2}
$$

where the spherical symmetry confines the particle orbits to a plane and we have chosen the $\theta=\pi / 2$ plane. This is the Newtonian expression for radial acceleration under gravity in polar coordinates.

Similarly the $\mu=3$ equation becomes $r \frac{d^{2} \phi}{d t^{2}}+2 \frac{d r}{d t} \frac{d \phi}{d t}=0$. This is the Newtonian expression for transverse acceleration in polar coordinates.

Problem 12 Expanding $1=g_{\mu \nu} u^{\mu} u^{\nu}$ gives for radial motion

$$
1=g_{00}\left(u^{0}\right)^{2}+g_{11}\left(u^{1}\right)^{2} .
$$

Substituting for the metric coefficients with $u^{0}=E /(1-2 m / r)$ gives

$$
1=\left(1-\frac{2 m}{r}\right) \frac{E^{2}}{(1-2 m / r)^{2}}-\frac{\left(u^{1}\right)^{2}}{(1-2 m / r)} .
$$

Finally, rearranging and taking square roots gives

$$
u^{1}= \pm\left[E^{2}-\left(1-\frac{2 m}{r}\right)\right]^{1 / 2} \text {. }
$$


Problem 13 The 4-velocity of an observer hovering at radius $r$ is

$$
u_{H}^{\mu}=\left(\left(1-\frac{2 m}{r}\right)^{-\frac{1}{2}}, 0,0,0\right) .
$$

The covariant components of the 4-velocity of a particle in radial free fall are

$$
\left(u_{\mu}\right)=\left(E, u_{1}, 0,0\right) .
$$

So the energy per unit mass of the falling particle in the frame of the observer is given by

$$
u_{H}^{\mu} u_{\mu}=\gamma=\left(1-v^{2}\right)^{-\frac{1}{2}} .
$$

where $v$ is the local velocity of the particle. Evaluating the scalar product in the Schwarzschild frame gives $E(1-2 m / r)^{-\frac{1}{2}}$. But the scalar product is an invariant, so we can equate it to its value $\gamma$ in a local inertial frame, hence

$$
E(1-2 m / r)^{-\frac{1}{2}}=\left(1-v^{2}\right)^{-\frac{1}{2}} .
$$

The velocity at $r$ of a particle falling from infinity is just the velocity needed to project the particle back from $r$ to infinity i.e. the escape velocity; hence setting $E=1$, the energy of a particle dropped from rest at infinity, gives

$$
v_{e s c}=\left(\frac{2 m}{r}\right)^{\frac{1}{2}} .
$$

At the horizon the escape velocity is the speed of light.

Problem 14 Doing this analytically is rather lengthy so an easier way is to plot the effective potential against $r$ (see figure 2) and note that the maximum occurs at the smaller value of $r$.

Problem 15 (a) Invert equation (2.26) to get $L$ in terms of $r$ and the result follows. (b) Substitute for $L$ in equation (2.19) and set $d r / d \tau=0$, the condition for a circular orbit.

Problem 16 From the geodesic equations (2.14) and(2.17)

$$
\frac{d t}{d \tau}=\frac{E}{\left(1-\frac{2 m}{r}\right)}
$$

and

$$
\frac{d \phi}{d \tau}=\frac{L}{r^{2}}
$$


where $\tau$ is the proper time of the orbiting particle. Substituting the expression for $L$ obtained in problem 15 into (3) and integrating over an orbit gives

$$
\tau=\frac{2 \pi r^{3 / 2}}{m^{1 / 2}}\left(1-\frac{3 m}{r}\right)^{\frac{1}{2}} .
$$

To obtain the period measured by a distant observer we divide (3) by (2):

$$
\frac{d \phi}{d t}=\frac{d \phi / d \tau}{d t / d \tau}=\frac{L(1-2 m / r)}{r^{2} E} .
$$

Now substitute for $E$ and $L$ the expressions obtained in problem 15 and integrate over one orbit to get

$$
T=\frac{2 \pi r^{\frac{3}{2}}}{m^{\frac{1}{2}}}
$$

Problem 17 The energy per unit mass of a particle in a circular orbit is

$$
E=\left(1-\frac{2 m}{r}\right)\left(1-\frac{3 m}{r}\right)^{-\frac{1}{2}} .
$$

Put $E=1$, the energy of a particle at rest at infinity, and solve for $r$ to get $r=4 \mathrm{~m}$. Inserting this value of $r$ into $L=(m r)^{\frac{1}{2}}(1-3 m / r)^{-\frac{1}{2}}$ gives $L=4 m$ thus $L / E=4 m$. This is an unstable orbit because it lies inside the last stable orbit at $r=6 \mathrm{~m}$. Note that in Newtonian physics a particle coming in from infinity must loose energy before it can be inserted into a circular orbit.

Problem 18 The velocity $v$ of a particle in a circular orbit at radius $r$ is related to the escape velocity $v_{\text {esc }}$ from that radius by

$$
v=\frac{v_{\mathrm{esc}}}{\sqrt{2}(1-2 m / r)^{1 / 2}} .
$$

Clearly when $r=4 m$ then $v=v_{\text {esc }}$. For $r<4 m$ the value of the denominator falls with decreasing $r$ and $v>v_{\text {esc }}$.

Problem 19 We evaluate $d \tau / m_{0}$ in a local inertial frame as we can use special relativity in such a frame. The infinitesimal time-like interval between a pair of events along the world line of a particle having velocity $u$ in flat spacetime is $d \tau=$ $d t\left(1-u^{2}\right)^{1 / 2}$. The mass $m_{0}$ of the particle is related to its energy and momentum by $m_{0}=\left(E^{2}-p^{2}\right)^{1 / 2}=E\left(1-u^{2}\right)^{1 / 2}$ as $p=E u$ : note that $E$ here is the energy of the particle not the energy per unit mass. So $d \tau / m_{0}=d t / E$. This expression is independent of the velocity of the particle so it also applies to photons. Hence $d \tau / m_{0} \rightarrow d t / E_{p h}$ which is finite. 
Problem 20 The 4 -velocity of a hovering observer at an arbitrary radial coordinate $r$ is $\left(u^{\mu}\right)=\left((1-2 m / r)^{-\frac{1}{2}}, 0,0,0\right)$ and the 4-momentum of a particle at $r$ has covariant components $\left(p_{\mu}\right)=\left(E, p_{1}, p_{2}, p_{3}\right)$. To obtain the velocity of the particle with respect to the local hovering observer we evaluate the scalar product $u^{\mu} p_{\mu}=\gamma m_{0}$ in the local frame and in the Schwarzschild frame and equate the results. This gives $m_{0} E(1-2 m / r)^{-\frac{1}{2}}=m_{0}\left(1-v^{2}\right)^{-\frac{1}{2}}$. Solving for $v$ the velocity of the particle gives

$$
v^{2}=\frac{m_{0}^{2} E^{2}-(1-2 m / r) m_{0}^{2}}{m_{0}^{2} E^{2}} .
$$

Now as $m_{0} \rightarrow 0, m_{0} E \rightarrow E_{p h}$ and the second term in the numerator goes to zero.Thus in the limit $v=1$, the speed of light. An alternative way to do this is to cancel the $m_{0}$ and remember that $E$ is the energy at infinity, so $E^{2}=\left(1-v_{\infty}^{2}\right)^{-1}$. Now $v^{2}=1-\left(1-v_{\infty}^{2}\right)(1-2 m / r)$. Set $v$ at infinity equal to the speed of light; then the local speed $v$ is everywhere equal to the speed of light.

Problem 21 This calculation follows closely the calculation in section 2.4.1 We evaluate the scalar product $u^{\mu} p_{\mu}=h \nu$, where $u$ is the 4 -velocity of the falling spaceship, $p$ the 4-momentum of a photon coming from infinity and $h \nu$ is the energy of the photon in the spaceship frame. Now we evaluate the scalar product in the Schwarzschild frame which is the frame of an observer at infinity and equate it to $h \nu$. The components of the probe's 4-velocity in the Schwarzschild frame are $\left(u^{\mu}\right)=\left(\left(1-\frac{2 m}{r}\right)^{-1},-\left(\frac{2 m}{r}\right)^{1 / 2}, 0,0\right)$ and the covariant components of momentum of the inwardly propagating photon are $\left(p_{\mu}\right)=\left(h \nu_{\infty}, h \nu_{\infty}\left(1-\frac{2 m}{r}\right)^{-1}, 0,0\right)$. So finally we get

$$
h \nu_{\infty}\left[1+\left(\frac{2 m}{r}\right)^{1 / 2}\right]^{-1}=h \nu
$$

The crew can continue to receive signals even when they are inside the horizon. Only as the singularity at $r=0$ is approached does the signal become infinitely redshifted.

Problem 22 The conditions for a circular orbit are $\frac{d r}{d \lambda}=0$ and $\frac{d^{2} r}{d \lambda^{2}}=0$. Equation (2.28) gives

$$
\left(\frac{d r}{d \lambda}\right)^{2}=E_{p h}^{2}-\frac{L_{p h}^{2}}{r^{2}}\left(1-\frac{2 m}{r}\right)
$$

Differentiating we get

$$
\frac{d^{2} r}{d \lambda^{2}}=\frac{L_{p h}^{2}}{r^{3}}\left(1-\frac{2 m}{r}\right)-\frac{2 m L_{p h}^{2}}{r^{4}}
$$

With $L_{p h} / E_{p h}=3 \sqrt{3} m$ both of these expressions are zero at $r=3 m$. So the light ray is indeed in a circular orbit at this radius. 
Problem 23 The apparent diameter of the hole is $3 \sqrt{3}\left(2 G M / c^{2}\right)=6.2 \times 10^{10} \mathrm{~m}$. So at the Earth it subtends an angle $\sim 51 \mu$ as.

Problem 24 We start from

$$
\left(\frac{d r}{d \phi}\right)^{2}=\left(\frac{d t / d \tau}{d \phi / d \tau}\right)^{2}=\frac{E^{2}-\left(1+L^{2} / r^{2}\right)(1-2 m / r)}{L^{2} / r^{4}}
$$

Let $u=1 / r$ then

$$
\frac{d u}{d \phi}=-\frac{1}{r^{2}} \frac{d r}{d \phi} .
$$

Changing to the new variable in the above expression gives

$$
\left(\frac{d u}{d \phi}\right)^{2}=\frac{E^{2}}{L^{2}}-\left(1+L^{2} u^{2}\right)(1-2 m u) / L^{2},
$$

which, on differentiating with respect to $\phi$, gives

$$
\frac{d^{2} u}{d \phi^{2}}+u=\frac{m}{L^{2}}+3 m u^{2}
$$

In the Newtonian limit the term $3 m u^{2}$ is zero so it represents the departure from Newtonian physics. Making the substitution $u=l^{-1}(1+\varepsilon \cos \lambda \phi)$ and collecting together the cosine terms gives

$$
\left(-\frac{\varepsilon \lambda^{2}}{l}+\frac{\varepsilon}{l}-\frac{6 m \varepsilon}{l^{2}}\right) \cos \lambda \phi+\frac{1}{l}=\frac{m}{L^{2}}+\frac{3 m}{l^{2}} .
$$

For a solution to this equation the coefficient of the $\cos \lambda \phi$ term must vanish as there is no $\phi$ on the right hand side; that is $\lambda=(1-6 m / l)^{1 / 2} \sim 1-3 m / l$ and $1 / l=m / L^{2}+3 m / l^{2}=m / L^{2}$. In the Newtonian limit $\lambda=1$ so here $3 m / l$ is small for weak fields. In one orbit $\lambda \phi=2 \pi$ so $\phi=2 \pi /(1-3 m / l) \approx 2 \pi+6 \pi m / l$. Therefore in one orbit $\phi$ has turned through the extra angle $6 \pi m / l=6 \pi m^{2} / L^{2}$. For a circular or near circular orbit $L^{2}=m r /(1-3 m / r)=m r$ for $r>>m$. So the amount of precession per orbit is $\Delta \phi=6 \pi m / r$. For the planet Mercury, for example, this comes to 43 seconds of arc per century. For a light ray

$$
\left(\frac{d r}{d \phi}\right)^{2}=\frac{E_{p h}-\left(L^{2} / r^{2}\right)(1-2 m / r)}{L^{2} / r^{4}}
$$

Setting $u=1 / r$ and differentiating gives

$$
\frac{d^{2} u}{d \phi^{2}}+u=3 m u^{2}
$$

In the absence of the term $3 m u^{2}$ the solution to the equation is of the form $u=$ $A \cos (\phi+\alpha)$, which is the equation of a straight line in polar coordinates. Finally setting $u=$ constant in the above equation gives the solution $1 / u=r=3 \mathrm{~m}$. 
Problem 25 (a) From the result of problem 12, $\frac{d r}{d \tau}=-(2 m / r)^{1 / 2}$ for a particle falling from rest at infinity. Separating the variables and integrating:

$$
\int_{0}^{T} d \tau=-\int_{2 m}^{0} \frac{r^{1 / 2}}{(2 m)^{1 / 2}} d r
$$

from which $T=4 m / 3$.

(b) Use $g_{\mu \nu} u^{\mu} u^{\nu}=1$. Inside the horizon this gives

$$
1=-\left(\frac{2 m}{r}-1\right) \dot{t}^{2}+\left(\frac{2 m}{r}-1\right)^{-1} \dot{r}^{2}-r^{2} \dot{\theta}^{2}-r^{2} \sin ^{2} \theta \dot{\phi}^{2}
$$

where the dot represents differentiation with respect to proper time. For this to be satisfied the positive term on the right hand side must be $\geq 1$. The equals sign will give the maximum proper time so

$$
\left(\frac{2 m}{r}-1\right)^{-1} \dot{r}^{2}=1
$$

And hence

$$
\frac{d r}{d \tau}= \pm\left(\frac{2 m}{r}-1\right)^{\frac{1}{2}}
$$

Taking the minus sign for inward motion and separating the variables gives

$$
\int_{0}^{T} d \tau=-\int_{2 m}^{0}(2 m / r-1)^{-\frac{1}{2}} d r .
$$

Making the substitution $r=2 m \cos ^{2} \theta$ and integrating gives $T=\pi m$.

(c) Paths of maximum proper time are geodesics.

(d) Use equation (2.19) and set $L=0$ and $E=0$ as the conditions for a particle to be at rest at the horizon. This gives

$$
\frac{d r}{d \tau}=-\left(\frac{2 m}{r}-1\right)^{\frac{1}{2}}
$$

which, as in part (b), integrates to $T=\pi m$. So an astronaut who wishes to survive for as long as possible inside the horizon should fire his rockets outside the horizon to reduce his speed as much as possible before crossing the horizon.

Problem 26 The time spent in pain is $\tau=\frac{2}{3}\left(\frac{\Delta r}{g_{E}}\right)^{\frac{1}{2}}$. To convert from geometric units to physical units use $t$ (in metres) $=c t$ (in seconds) and $g\left(\right.$ metres $\left.^{-1}\right)=$ 
$c^{-2} g\left(\mathrm{~ms}^{-2}\right)$. On making these substitutions the $c$ 's cancel so the original expression is valid. Inserting $\Delta r=2 m$ and $g_{E}=9.81 \mathrm{~ms}^{-2}$ into the expression above gives $\tau=0.3 \mathrm{~s}$. The threshold of pain radius is $r_{p}=\left(\frac{2 m \Delta r}{g_{E}}\right)^{\frac{1}{3}}$ in geometric units. Converting to physical units this becomes $r_{p}=\left(\frac{2 G M \Delta r}{g_{E}}\right)^{\frac{1}{3}}$, after converting $g$ and setting $m=G M / c^{2}$. For $M=$ one solar mass $r_{p}=3.8 \times 10^{3} \mathrm{~km}$. This is well beyond the horizon of a one solar mass black hole, which is about $3 \mathrm{~km}$. The threshold of pain equals the Schwarzschild radius when $r_{p}=r_{s}$. This condition is realised when $M=\frac{c^{3}}{2 G}\left(\frac{\Delta r}{g}\right)^{1 / 2}=4.6 \times 10^{4}$ solar masses.

Problem 27 From the answer to problem 22 the time from crossing the horizon to hitting the singularity for a particle falling from a large distance is $4 \mathrm{~m} / 3$. Converting this expression to physical units and inverting to give mass in terms of time $T$ gives

$$
M=\frac{3 c^{3} T}{4 G} .
$$

For $T=1$ yr we get $M=5 \times 10^{12}$ solar masses. Note that only in the last 0.3 seconds will serious deformation occur, but $0.3 \mathrm{~s}$ is too short a time in which to suffer any pain.

Problem 28 In Rindler coordinates the metric is $d \tau^{2}=\kappa^{2} \xi^{2} d t^{2}-d \xi^{2}$. Transforming to the coordinates $(T, X)$ where

$$
\begin{aligned}
T & =\xi \sinh \kappa t \\
X & =\xi \cosh \kappa t \\
d T & =d \xi \sinh \kappa t+d t \kappa \xi \cosh \kappa t \\
d X & =d \xi \cosh \kappa t+d t \kappa \xi \sinh \kappa t .
\end{aligned}
$$

Squaring and adding gives

$$
d T^{2}-d X^{2}=\kappa^{2} \xi^{2} d t^{2}-d \xi^{2}
$$

So

$$
d \tau^{2}=d T^{2}-d X^{2}
$$

Problem 29 On the curve $\xi=1 / a=$ constant

$$
\begin{aligned}
T & =a^{-1} \sinh \kappa t \\
X & =a^{-1} \cosh \kappa t .
\end{aligned}
$$

Differentiating $T$ and $X$ with respect to $\tau$ gives

$$
\frac{d T}{d \tau}=a^{-1} \kappa \cosh \kappa t \frac{d t}{d \tau}
$$


and

$$
\frac{d X}{d \tau}=a^{-1} \kappa \sinh \kappa t \frac{d t}{d \tau}
$$

Differentiating these expressions again with respect to $\tau$ gives the two non zero components of the 4-acceleration:

$$
\begin{aligned}
\frac{d^{2} T}{d \tau^{2}} & =a^{-1} \kappa^{2} \sinh \kappa t\left(\frac{d t}{d \tau}\right)^{2}+a^{-1} \kappa \cosh \kappa t \frac{d^{2} t}{d \tau^{2}} \\
\frac{d^{2} X}{d \tau^{2}} & =a^{-1} \kappa^{2} \cosh \kappa t\left(\frac{d t}{d \tau}\right)^{2}+a^{-1} \kappa \sinh \kappa t \frac{d^{2} t}{d \tau^{2}}
\end{aligned}
$$

Now from the Rindler metric with $\xi=1 / a=$ constant

$$
\left(\frac{d t}{d \tau}\right)^{2}=\frac{1}{\kappa^{2} \xi^{2}}=\frac{a^{2}}{\kappa^{2}} \quad \text { and } \quad \frac{d^{2} t}{d \tau^{2}}=0
$$

So the proper acceleration $A^{\mu}=(a \sinh \kappa \tau, a \cosh \kappa \tau, 0,0)$ and $A^{\mu} A_{\mu}=-a^{2}$. So the magnitude of the proper acceleration is $a$.

Problem 30 We wish to find the limiting value of $\cos \psi$ as $r \rightarrow 0$, where

$$
\cos \psi=\frac{\left[1-(1-2 m / r) b^{2} / r^{2}\right]^{1 / 2}-(2 m / r)^{1 / 2}}{1-(2 m / r)^{1 / 2}\left[1-(1-2 m / r) b^{2} / r^{2}\right]^{1 / 2}}
$$

The velocity of a particle falling from rest at infinity, $(2 \mathrm{~m} / r)^{1 / 2}$, has been inserted into equation (2.52). As $r \rightarrow 0$ the term $(1-2 m / r) b^{2} / r^{2}$ dominates so

$$
\cos \psi \rightarrow \frac{\left[(1-2 m / r) b^{2} / r^{2}\right]^{1 / 2}}{(2 m / r)^{1 / 2}\left[1-(1-2 m / r) b^{2} / r^{2}\right]^{1 / 2}} \rightarrow-\left(\frac{r}{2 m}\right)^{1 / 2}
$$

Problem 31 From section 3.2 the event horizon is a surface of constant $r$ and constant $t$ but its equatorial and polar circumferences are not the same. From section 3.2.1 the equatorial circumference of an extreme Kerr black hole is $2 \pi\left(r_{+}^{2}+\right.$ $\left.m^{2}\right) / r_{+}=4 \pi m$ as $r_{+}=m$. From the metric, the polar circumference of this black hole is $\int_{0}^{2 \pi} \rho d \theta=\int_{0}^{2 \pi}\left(r_{+}^{2}+m^{2} \cos ^{2} \theta\right)^{\frac{1}{2}} d \theta=m \int_{0}^{2 \pi}\left(1+\cos ^{2} \theta\right)^{\frac{1}{2}} d \theta$. This integral cannot be evaluated in terms of elementary functions. However we can estimate its value as follows. Expanding $\left(1+\cos ^{2} \theta\right)^{\frac{1}{2}}$ by the binomial theorem gives $1+\frac{1}{2} \cos ^{2} \theta-\frac{1}{8} \cos ^{4} \theta+\frac{1}{16} \cos ^{6} \theta+\ldots$ for the integrand. We can evaluate these four integrals: they give respectively $2 \pi+\pi / 2-3 \pi / 16+5 \pi / 48=2.42$. So the circumference is $\sim 2.42 \pi m=7.6 \mathrm{~m}$. This is less than the equatorial circumference. 
Problem 32 Putting $m=0$ into the Boyer Lindquist form of the Kerr metric gives

$$
d s^{2}=d t^{2}-\frac{\rho^{2}}{r^{2}+a^{2}} d r^{2}-\rho^{2} d \theta^{2}-\left(r^{2}+a^{2}\right) \sin ^{2} \theta d \phi^{2} .
$$

We have to show that the coordinate transformation given in the question changes the flat space metric $d x^{2}+d y^{2}+d z^{2}$ into the spatial part of the metric given above. Differentiating the $x$ coordinate transformation gives

$$
d x=\left(r^{2}+a^{2}\right)^{-\frac{1}{2}} r \sin \theta \cos \phi d r+\left(r^{2}+a^{2}\right)^{\frac{1}{2}} \cos \theta \cos \phi d \theta-\left(r^{2}+a^{2}\right)^{-\frac{1}{2}} \sin \theta \sin \phi d \phi .
$$

So

$$
\begin{aligned}
d x^{2}= & \left(r^{2}+a^{2}\right)^{-1} r^{2} \sin ^{2} \theta \cos ^{2} \phi d r^{2}+\left(r^{2}+a^{2}\right) \cos ^{2} \theta \cos ^{2} \phi d \theta^{2} \\
& +\left(r^{2}+a^{2}\right) \sin ^{2} \theta \sin ^{2} \phi d \phi^{2}+2 \cos \theta \sin \theta \cos ^{2} \phi r d r d \theta \\
& -2 \sin ^{2} \theta \cos \phi \sin \phi r d r d \phi-2\left(r^{2}+a^{2}\right) \cos \theta \sin \theta \cos \phi \sin \phi d \theta d \phi .
\end{aligned}
$$

Proceeding in the same way with the $y$ and $z$ transformations and adding them together gives finally

$$
d x^{2}+d y^{2}+d z^{2}=\frac{\rho^{2}}{\left(r^{2}+a^{2}\right)}+\rho^{2} d \theta^{2}+\left(r^{2}+a^{2}\right) \sin ^{2} \theta d \phi^{2} .
$$

Problem 33 We consider the identity $g_{03}^{2}-g_{00} g_{03}=\Delta \sin ^{2} \theta$. Substituting for the metric coefficients and extracting the common factor gives

$$
\frac{\sin ^{2} \theta}{\rho^{4}}\left[4 m^{2} a^{2} r^{2} \sin ^{2} \theta+\left(\Delta-a^{2} \sin ^{2} \theta\right) A\right] .
$$

Inserting the expressions for $A$ and $\Delta$ and using $\rho^{2}=r^{2}+a^{2} \cos ^{2} \theta$ gives

$$
\frac{\sin ^{2} \theta}{\rho^{4}}\left[4 m^{2} a^{2} r^{2} \sin ^{2} \theta+\left(\rho^{2}-2 m r\right)\left(\rho^{2}\left(r^{2}+a^{2}\right)+2 m a^{2} r \sin ^{2} \theta\right)\right] .
$$

Finally the expression inside the square brackets reduces to $\rho^{4} \Delta$ so the left hand side becomes $\Delta \sin ^{2} \theta$ as required. The other identities follow in a similar manner.

Problem 34 For $r>>a$

$$
\begin{aligned}
& g_{00}=\frac{\left(\Delta-a^{2} \sin ^{2} \theta\right)}{\rho^{2}} \rightarrow \frac{\Delta}{r^{2}} \rightarrow\left(1-\frac{2 m}{r}\right) \\
& g_{03}=\frac{\left(2 m a r \sin ^{2} \theta\right)}{\rho^{2}} \rightarrow \frac{\left(2 m a \sin ^{2} \theta\right)}{r} \\
& g_{22}=-\rho^{2} \rightarrow-r^{2} \\
& g_{33}=-\frac{A \sin ^{2} \theta}{\rho^{2}} \rightarrow-r^{2} \sin ^{2} \theta .
\end{aligned}
$$

With these values of the metric coefficients the Kerr metric takes the form in equation (3.11). 
Problem 35 The radius of the event horizon is $r_{+}=m+\left(m^{2}-a^{2}\right)^{1 / 2}$. If $a>m$ then $r_{+}$becomes complex so there is no event horizon.

Problem 36 At the turning points of a bound orbit $d \theta / d \tau=0$, so multiplying equation (3.16) through by $\sin ^{2} \theta_{T P}$ gives

$$
Q \sin ^{2} \theta_{T P}=\left(1-\sin ^{2} \theta_{T P}\right) a^{2}\left(1-E^{2}\right) \sin ^{2} \theta_{T P}+\left(1-\sin ^{2} \theta_{T P}\right) L_{z}^{2} .
$$

Rearranging gives a quadratic equation in $\sin ^{2} \theta_{T P}$

$$
a^{2}\left(1-E^{2}\right) \sin ^{4} \theta_{T P}+\left[Q-a^{2}\left(1-E^{2}\right)+L_{z}^{2}\right] \sin ^{2} \theta_{T P}-L_{z}^{2}=0
$$

The solution of which is

$\sin ^{2} \theta_{T P}=\frac{-\left(Q-a^{2}\left(1-E^{2}\right)+L_{z}^{2}\right) \pm\left[\left(Q-a^{2}\left(1-E^{2}\right)+L_{z}^{2}\right)^{2}+4 a^{2}\left(1-E^{2}\right) L_{z}^{2}\right]^{\frac{1}{2}}}{2 a^{2}\left(1-E^{2}\right)}$.

For a bound orbit $(E<1)$ the term inside the square root is larger than the first term in the numerator, so to ensure that $\sin ^{2} \theta_{T P}$ is positive we take the plus sign. On taking the square root we see that there are two real values of $\sin \theta_{T P}$, that is two turning points for a bound orbit. Note that $Q=0$ gives $\sin ^{2} \theta_{T P}=1$ : this is an orbit in the equatorial plane. The condition $L_{z}=0$ gives $\sin ^{2} \theta_{T P}=0$, which is an orbit with turning points at $\theta=0$ and $\theta=\pi$ i.e. an orbit that passes over the two poles.

Problem 37 We have $\omega=\frac{u^{3}}{u^{0}}=\frac{g^{30} u_{0}+g^{33} u_{3}}{g^{00} u_{0}+g^{03} u_{3}}=\frac{g^{30}}{g^{00}}$ where the second equality follows because $L=-u_{3}=0$. Also from $u_{3}=g_{03} u^{0}+g_{33} u^{3}$ we get

$$
\frac{u^{3}}{u^{0}}=\omega=-\frac{g_{03}}{g_{33}} .
$$

Problem 38 The circumference of the event horizon of a Kerr black hole is $4 \pi m$. (See section 3.2.1) Now velocity $=$ circumference/period $=\frac{4 \pi m}{2 \pi / \omega}$. From equation (3.29)3.29 $\omega=1 /(2 m)$ for an extreme black hole. Hence relative to an observer at infinity the velocity $=1$ in geometric units, which is the speed of light.

Problem 39 From equation (3.29) the angular velocity of a Kerr black hole is given by $\omega_{+}=\frac{a}{2 m r_{+}}$. For $a<<m, r_{+} \sim 2 m$ so $\omega_{+} \sim a /\left(4 m^{2}\right)$. By the usual definition, the moment of inertia $I$ is given by $I=j / \omega_{+}$. So putting $j=m a$ and substituting for $\omega_{+}$gives $I=4 m^{3}$.

Problem 40 For a particle released from rest at infinity with zero angular momentum $d \theta / d \tau$ is given by equation (3.18). Setting $E=1$ gives $d \theta / d \tau=0$. From equation (3.16) $d \phi / d \tau \neq 0$ so the particle moves inwards on a conical spiral of constant half angle. 
Problem 41 Setting $a=0$ in equation (3.37): $\left(\frac{g_{33}}{-\Delta \sin ^{2} \theta}\right)^{1 / 2}=\left(1-\frac{2 m}{r}\right)^{-1 / 2}$ and $\omega=0$. So the components of $\left(u_{Z A M O}^{\mu}\right)$ become $\left((1-2 m / r)^{-1 / 2}, 0,0,0\right)$ which are the components of a hovering observer in the Schwarzschild metric.

Problem 42 The 4-velocity of a hovering observer is $\left(u^{\mu}\right)=\left(g_{00}^{-1 / 2}, 0,0,0\right)$ so the covariant components are

$$
\left(u_{\mu}\right)=\left(g_{00}^{1 / 2}, 0,0, g_{03} g_{00}^{-1 / 2}\right)
$$

where $u_{3}=g_{03} u^{0}$ has been used. Now evaluate the scalar product $u_{Z A M O}^{\mu} u_{\mu}$ in the Schwarzschild frame and equate it to the energy per unit mass in a local frame. Using equation (3.37) for $u_{Z A M O}^{\mu}$ with $\theta=\pi / 2$ we get

$$
\left(\frac{g_{33}}{-\Delta}\right)^{\frac{1}{2}}\left(g_{00}^{1 / 2}+\omega g_{03} g_{00}^{-1 / 2}\right)=\gamma
$$

Inserting $\omega=-g_{03} / g_{33}$ and using the identity $g_{03}^{2}-g_{00} g_{33}=\Delta \sin ^{2} \theta$ we get

$$
\left(\frac{-\Delta}{g_{33} g_{00}}\right)^{\frac{1}{2}}=\gamma=\left(1-\nu^{2}\right)^{-\frac{1}{2}}
$$

or

$$
\nu^{2}=1+\frac{g_{33} g_{00}}{\Delta}
$$

Substituting for $g_{33}$ and $g_{00}$ and simplifying gives

$$
\nu=\frac{2 m a}{r \Delta^{1 / 2}} .
$$

Problem 43 For purely radial motion of light the metric becomes

$$
0=g_{00}\left(\frac{d t}{d \lambda}\right)^{2}+g_{11}\left(\frac{d r}{d \lambda}\right)^{2} .
$$

But inside the static limit both the metric coefficients are negative so the condition cannot be satisfied.

Problem 44 Let $u^{\mu}$ be the 4-vector of the stationary particle. Then the 3-component of $u_{\mu}=g_{\mu \nu} u^{\nu}$ is

$$
u_{3}=g_{03} u^{0}+g_{33} u^{3}=g_{03} u^{0}
$$

as $u^{3}=d \phi / d \tau=0$. Now, from the metric, the 4-velocity of a stationary particle is

$$
u^{\mu}=\left(g_{00}^{-1 / 2}, 0,0,0\right)
$$


So $u_{3}=-L_{z}=g_{30} g_{00}^{-1 / 2}$. Finally

$$
L_{z}=-\frac{2 m a r \sin ^{2} \theta}{\rho\left(\Delta-a^{2} \sin ^{2} \theta\right)^{1 / 2}} .
$$

The denominator is zero at the static limit surface so $L_{z} \rightarrow \infty$ there. Therefore only a photon can remain static at this location. Inside the static limit surface the angular momentum becomes imaginary so a particle cannot be at rest.

Problem 45 Equation (3.49) written as a quadratic in $a$ is

$$
3 a^{2} \mp 8 m^{\frac{1}{2}} r_{m s}^{\frac{1}{2}} a-\left(r_{m s}^{2}-6 m r_{m s}\right)=0
$$

so

$$
a=\frac{r_{m s}^{\frac{1}{2}} m^{\frac{1}{2}}}{3}\left( \pm 4 \mp\left(\frac{3 r_{m s}}{m}-2\right)^{\frac{1}{2}}\right) .
$$

To check the signs set $r_{m s}=m$. This gives $a=m$ ( the prograde case) with first sign + and second sign - With $r_{m s}=9 m$ we get $a=m$ (the retrograde case) with the two signs reversed. So for a retrograde orbit with $r_{m s}=7.5 m, a=0.48$ as required. For a prograde orbit with $r_{m s}=4.12 m, a=0.53 m$.

Problem 46 We are given $r=4.12 m$ for the last stable orbit of a black hole with $a=0.53 m$ and $T=2 \pi\left(\frac{r^{3 / 2}}{m^{1 / 2}} \pm a\right)$. Substituting for $r$ and $a$ gives $T=$ $2 \pi m\left[(4.12)^{3 / 2}+0.53\right]$. Putting $m=G M / c^{2}$ to convert to physical units and using $M=3.7 \times 10^{6} M_{\odot}$ gives $T=17.1$ minutes.

Problem 47 We use the expression for $a$ as a function of $r_{m s}$ derived in problem 45 to obtain values of $r_{m s}$ for a range of values of $a$. We then use these values of $r$ in equation (3.48).

Problem 48 Equation (3.44), after substitution for $E^{2}$ from equation (3.48), becomes

$$
L^{2}=\frac{2 m}{3 r_{m s}}\left(3 r_{m s}^{2}-a^{2}\right)
$$

Now solving equation (3.49) as a quadratic in $a$ (see problem 45) gives

$$
a=\frac{m^{\frac{1}{2}} r_{m s}^{\frac{1}{2}}}{3}\left[ \pm 4+\left(3 \frac{r_{m s}}{m}-2\right)^{\frac{1}{2}}\right]
$$

Squaring $a$ and substituting into (4) gives

$$
L^{2}=\left(\frac{4 m^{2}}{27}\right)\left[12\left(\frac{r_{m s}}{m}\right)-7+4\left(3 \frac{r_{m s}}{m}-2\right)^{\frac{1}{2}}\right] .
$$


Finally taking the square root gives

$$
L= \pm \frac{2 m}{3 \sqrt{3}}\left[1+2\left(3 \frac{r_{m s}}{m}-2\right)^{\frac{1}{2}}\right]
$$

Problem 49 (a) For the co-rotating case in the extreme Kerr limit $r_{m s}=m$ so $L=2 m / \sqrt{3}$.

(b) For the counter-rotating case $r_{m s}=9 m$ so $L=-22 m /(3 \sqrt{3})$.

Problem 50 A direct substitution of $a=0$ and $r=3 m$ gives $b=0 / 0$ which is indeterminate. Instead use the expression in the hint:

$$
(r / m)^{\frac{3}{2}}-3(r / m)^{\frac{1}{2}} \pm 2(a / m)=0
$$

Solving for $a$ we get $a=\mp(r / m)^{1 / 2}(r-3 m) / 2$. Now substituting this value for $a$ into the expression for $b$ gives $b=(r / m)^{1 / 2}(r+3 m) / 2$. But $r=3 m$ so $b=3 \sqrt{3} m$.

Problem 51 For large $r$ equation (3.53) becomes

$$
E^{2}=\frac{r\left(r^{2}-2 m r\right)^{2}}{r^{2}\left(r^{3}-3 m r^{2}\right)}=\frac{\left(1-\frac{2 m}{r}\right)^{2}}{\left(1-\frac{3 m}{r}\right)}
$$

which is the Schwarzschild result for the energy of a particle in a circular polar orbit.

Problem 52 A plot of energy per unit mass $E$ against $r$ for a circular polar orbit shows that the minimum value of the specific energy is 0.937 at $r \sim 5.3 \mathrm{~m}$. Therefore the maximum binding energy is $1-0.937=0.063$ or $6.3 \%$ of the rest mass of a particle.

Problem 53 (a) In geometrical units $\omega_{p}=2 m a / r^{3}$. Now $c \times t$ (seconds) $=\mathrm{t}$ (metres) so $\omega\left(\right.$ seconds $\left.^{-1}\right)=\mathrm{c} \times \omega\left(\right.$ metres $\left.^{-1}\right)$. We also have $m=G M / c^{2}$ and $a=J / M c$. So

$$
\omega\left(\mathrm{s}^{-1}\right)=\frac{2 G J}{c^{2} r^{3}} .
$$

(b) We have

$$
\omega_{p}=\frac{2 m a}{r^{3}} \times \frac{m^{3}}{m^{3}}=\frac{2 a}{m^{2}(r / m)^{3}},
$$

so for a given $r / m$ the precessional frequency is inversely proportional to the mass of the black hole squared. 
Problem 54 For a polar orbit about the Earth we can use the expression for the precession angular velocity given in SI units in problem 53

$$
\frac{d \phi}{d t}=\frac{2 G J}{c^{2} r^{3}}
$$

with $J=0.33 M_{\oplus} R_{\oplus}^{2} \Omega_{\oplus}=0.33 \times 6 \times 10^{24} \times\left(6.37 \times 10^{6}\right)^{2} \times 7.3 \times 10^{-5}=5.86 \times 10^{33} \mathrm{Js}$. So in one year $\Delta \phi=\left(2 \times 6.7 \times 10^{-11} \times 5.86 \times 10^{33} \times 3.16 \times 10^{7}\right) /\left(9 \times 10^{16} \times\left(12 \times 10^{6}\right)^{3}\right)=$ $1.6 \times 10^{-7}$ radians or 33 milli-arc seconds.

Problem 55 For a polar orbit $L_{z}=0$ so equation (3.16) gives for the polar orbit

$$
\rho^{2} \frac{d \phi}{d \tau}=\frac{2 \operatorname{mar} E}{\Delta}
$$

For a zero angular momentum particle in the equatorial plane we have $L_{z}=0$ and $\theta=\pi / 2$ so for this case equation (3.16) gives

$$
r^{2} \frac{d \phi}{d \tau}=\frac{2 m a r E}{\Delta}
$$

When $r \gg a$ we have $\rho \rightarrow r$ and these two expressions tend to the same value.

Problem 56 The condition $V_{r}=0$ gives

$$
Q=\left[E^{2}\left(r^{2}+a^{2}\right)^{2}-\Delta a^{2} E^{2}-r^{2} \Delta\right] / \Delta
$$

Substituting for $E^{2}$ from equation (3.53) gives, after simplification, the required expression. The final part of the question follows immediately on setting $a=0$. In this limiting case $Q=L^{2}$.

Problem 57 The Killing vector $\left(k^{\mu}\right)=(1,0,0,0)$ has covariant components $k_{\mu}=$ $g_{\mu \nu} k^{\nu}$ so $k_{0}=g_{00} k^{0}+g_{03} k^{3}=g_{00} k^{0}$ as $k^{3}=0$. So the scalar product $k^{\mu} k_{\mu}=g_{00}$. At the static limit surface $g_{00}$ changes from being positive outside to negative inside. Therefore the vector $k^{\mu}$ is spacelike inside the static limit surface.

Problem 58 Setting the index $\mu=0$ in $p_{\mu}=g_{\mu \nu} p^{\nu}$ for a photon gives

$$
p_{0}=E_{p h}=g_{00} p^{0}+g_{03} p^{3}=g_{00} \frac{d t}{d \lambda}+g_{03} \frac{d \phi}{d \lambda}
$$

or

$$
E_{p h}=\frac{d t}{d \lambda}\left(g_{00}+g_{03} \Omega\right)
$$

where $\Omega=\frac{d \phi}{d t}$. Now from equation (3.40) $\Omega=\omega \pm \frac{r^{2} \Delta^{1 / 2}}{A}$ for a tangentially projected photon and $\omega=-\frac{g_{03}}{g_{33}}$. Substituting these expressions into (8) and using the identity $g_{03}^{2}-g_{00} g_{33}=\Delta \sin ^{2} \theta$ gives for motion in the equatorial plane

$$
E_{p h}=\frac{d t}{d \lambda}\left(\frac{\Delta r^{2} \pm 2 \operatorname{mar} \Delta^{1 / 2}}{A}\right) .
$$


The time-like component of $p^{\mu}=g^{\mu \nu} p_{\nu}$ is $\frac{d t}{d \lambda}$ :

$$
\frac{d t}{d \lambda}=\frac{A E_{p h}-2 \operatorname{mar} L_{p h, z}}{r^{2} \Delta}
$$

Substituting into (9) and collecting the terms containing $E_{p h}$ on the left hand side gives, after some cancellations,

$$
E_{p h}=\frac{2 \operatorname{mar} L_{p h}+\left|L_{p h}\right| r^{2} \Delta^{1 / 2}}{A}
$$

where $L_{p h}$ is positive for co-rotation and negative for contra-rotation. A particle at the horizon $(\Delta=0)$ has $E_{p h}=\frac{2 m a r_{+} L_{p h, z}}{\left(2 m a r_{+}\right)^{2}}=\frac{a L_{p h, z}}{2 m r_{+}}=\omega_{+} L_{p h, z}$. Inserting $\dot{r}=0$ into equation (3.56) and setting the $r^{2} \Delta$ term inside the square root to zero, as we are treating a photon, reduces (3.56), after some simplification, to equation (10). If $\mathrm{L}$ is negative then equation (10) tells us that inside the ergosphere, where $r^{2} \Delta^{1 / 2}<2$ mar, $E_{p h}$ is negative.

Problem 59 The innermost stable orbit for a co-rotating particle around an extreme Kerr black hole is at $r_{m s}=m$. The angular momentum gained through accretion of a mass $d m$ from the last stable orbit of an extreme black hole, from equation (3.64) with $x_{m s}=1$, is given by $d j=2 m d m$. Integrating gives $j=m^{2}$.

Problem 60 From equation (3.68) $r_{m s} m=$ constant, so $9 m_{K}^{2}=6 m_{S}^{2}$ hence $m_{K}=$ $\sqrt{2 / 3} m_{s}$. So the fractional increase in mass of the black hole is $\sqrt{3 / 2}-1=22.5 \%$. To find the quantity of rest mass that has been accreted we employ the method used above. Equation (3.63) with $r_{m s}=9 m_{K}^{2} / m$ gives

$$
d m_{0}=\frac{d m}{\left(1-\frac{2 m^{2}}{27 m_{K}^{2}}\right)^{\frac{1}{2}}}
$$

Now integrating

$$
\Delta m=a \int_{m_{K}}^{\sqrt{3 / 2} m_{K}} \frac{d m}{\left(a^{2}-m^{2}\right)^{1 / 2}}
$$

where $a=\sqrt{27 / 2} m_{K}$. Evaluating the integral by using the substitution $m=a \sin \theta$ gives

$$
\Delta m_{0}=\left(\frac{27}{2}\right)^{\frac{1}{2}}\left[\sin ^{-1}\left(\frac{1}{3}\right)-\sin ^{-1}\left(\frac{2}{27}\right)^{\frac{1}{2}}\right] m_{K}
$$

so $\Delta m_{0}=0.236 m_{K}$. This is larger than the increase in the mass of the black hole by $0.011 m_{K}$. So $4.7 \%$ of the rest mass accreted has been radiated away. Thus, a black hole accreting from a contra-rotating disc will not be as luminous as one accreting from a co-rotating disc. 
Problem 61 To answer this question we have to show that the integral of $d s$ is positive around the circle $r=a \delta, T=$ constant in the $Z=0$ plane. Inserting these conditions into the metric in Kerr-Schild coordinates gives

$$
d s^{2}=-d X^{2}-d Y^{2}-\frac{2 m a^{3} \delta^{3}}{(a \delta)^{4}}\left[\frac{-a \delta(X d X+Y d Y)-a(X d Y-Y d X)}{(a \delta)^{2}+a^{2}}\right]^{2} .
$$

From the coordinate transformations in section 3.18 we get, with $\theta=\pi / 2$ :

$$
\begin{aligned}
d X & =(-r \sin \tilde{\phi}+a \cos \tilde{\phi}) d \tilde{\phi}=-Y d \tilde{\phi} \\
d Y & =(r \cos \tilde{\phi}+a \sin \tilde{\phi}) d \tilde{\phi}=X d \tilde{\phi}
\end{aligned}
$$

Substituting these results into (11) gives

$$
d s^{2}=\left[-r^{2}-a^{2}-\frac{2 m}{\delta}\left[\frac{-X^{2}-Y^{2}}{(a \delta)^{2}+a^{2}}\right]^{2}\right] d \tilde{\phi}^{2} .
$$

With delta negative and small $d s^{2}$ is positive and so the interval is timelike.

Problem 62 The event horizon is given by $\Delta=r^{2}+a^{2}+q^{2}-2 m r=0$. Solving this quadratic in $r$ for the radius of the horizon gives $r_{+}=m \pm\left(m^{2}-a^{2}-q^{2}\right)^{1 / 2}$. So $r_{+}$ is real only when $m^{2}>a^{2}+q^{2}$.

Problem 63 (i) We evaluate the scalar product $X^{\mu} X_{\mu}$, where $\left(X^{\mu}\right)=(1,0,0, \omega)$. Now $X_{0}=g_{00} X^{0}+g_{03} X^{3}$ and $X_{3}=g_{30} X^{0}+g_{33} X^{3}$ so $X_{\mu}=\left(g_{00}+g_{03} \omega, 0,0, g_{30}+g_{33} \omega\right)$ and $X^{\mu} X_{\mu}=g_{00}+g_{03} \omega+g_{03} \omega+g_{33} \omega^{2}$. Now $\omega=-g_{03} / g_{33}$ so $X^{\mu} X_{\mu}=-\left(\Delta \sin ^{2} \theta\right) / g_{33}$. But at the horizon $\Delta=0$, so $X^{\mu}$ is null at the horizon.

(ii) The metric in ingoing Eddington-Finkelstein coordinates is

$$
d s^{2}=\left(1-\frac{2 m}{r}\right) d v^{2}+2 d v d r-r^{2} d \tilde{\omega}^{2} .
$$

$X^{\mu}=(1,0,0,0)$ is null on the horizon so $X^{\nu}\left(\nabla_{\nu} x^{\mu}\right)=\kappa X^{\mu}$ reduces to

$$
\nabla_{0} X^{0}=\kappa
$$

Now $\nabla_{\nu} X^{\mu}=X_{, \nu}^{\mu}+\Gamma_{\nu \rho}^{\mu} X^{\rho}$ so $\nabla_{0} X^{0}=\Gamma_{00}^{0} X^{0}=\kappa$. From the definition of $\Gamma_{\nu \rho}^{\mu}$ (see equation (1.10)) with $g_{00}=(1-2 m / r)$ and $g_{01}=1 \Gamma_{00}^{0}=\frac{1}{2} g^{01} \frac{\partial g_{00}}{\partial r}=\frac{m}{r^{2}}$. At the horizon, $r=2 m$ so finally $\kappa=1 /(4 m)$. 
Problem 64 To verify that $m=\kappa A_{h} / 4 \pi+2 \omega_{+} j$. Substituting for $\kappa, A_{h}$ and $j$ :

$$
\kappa A_{h} / 4 \pi+2 \omega_{+} j=\frac{\left(m^{2}-a^{2}\right)^{1 / 2}}{2 m r_{+}} 2 m\left[m+\left(m^{2}-a^{2}\right)^{1 / 2}\right]+\frac{2 m a^{2}}{2 m r_{+}}
$$

which simplifies to

$$
\frac{m\left(m^{2}-a^{2}\right)^{1 / 2}}{r_{+}}+\frac{m^{2}}{r_{+}}=\frac{m\left[m+\left(m^{2}-a^{2}\right)^{1 / 2}\right]}{r_{+}}=m .
$$

This verifies Smarr's formula.

Problem 65 The solution to this problem follows closely the treatment for the increase in surface area due to the capture of a massive particle given in section 4.2.1. Equation (4.7) gives

$$
\delta A_{h}=\frac{16 m r_{+} \pi}{\left(m^{2}-a^{2}\right)^{1 / 2}}\left(\delta m-\omega_{+} \delta j\right) .
$$

For a photon $\delta m=E_{p h}$ and from section 3.10 .3 the impact parameter for a photon in a circular orbit around an extreme Kerr black hole is $b=L_{p h} / E_{p h}$ but $b=2 m$ for such a photon so $L_{p h} / E_{p h}=2 m$ and the change in angular momentum $\delta j=2 m E_{p h}$. Using these results the change in area becomes

$$
\delta A_{h}=\frac{16 m \pi E_{p h}}{\left(m^{2}-a^{2}\right)^{1 / 2}}\left(r_{+}-a\right)=\frac{16 m \pi E_{p h}}{\left(m^{2}-a^{2}\right)^{1 / 2}}\left[\left(m^{2}-a^{2}\right)^{1 / 2}+\varepsilon\right]
$$

as $r_{+}-a=\left(m^{2}-a^{2}\right)^{1 / 2}+\varepsilon$. (See section 4.2.1.) So finally the change in area as $a \rightarrow m$ is

$$
\delta A_{h}=16 m \pi E_{p h}
$$

So the area increases.

Problem 66 Starting from equation (4.5) $A_{h}=8 \pi m\left[m+\left(m^{2}-a^{2}\right)^{1 / 2}\right]$, rearrange and square to get $\left(\frac{A_{h}}{8 \pi m}-m\right)^{2}=m^{2}-a^{2}$. Evaluating the left hand side and putting $a=j / m$ gives $\left(\frac{A_{h}}{8 \pi m}\right)^{2}+\frac{j^{2}}{m^{2}}=\frac{2 A_{h}}{8 \pi}$. Finally multiply though by $4 \pi m^{2} / A_{h}$ to get $\frac{A_{h}}{16 \pi}+\frac{4 \pi j^{2}}{A_{h}}=m^{2}$.

Problem 67 From section 4.2.4 $m_{\text {rot }}=j^{2}\left(A_{h} / 4 \pi\right)^{-3 / 2}, j=I \omega_{+}$and for $a<<m$, $A_{h} \sim 16 \pi m^{2}$ so $m_{\text {rot }}=j^{2}\left(A_{h} / 4 \pi\right)^{-3 / 2}=I^{2} \omega^{2} / 8 m^{3}=\left(I / 8 m^{3}\right) I \omega_{+}^{2}=\frac{1}{2} I \omega_{+}^{2}$.

Problem 68 The total area of the event horizons of the two Schwarzschild black holes before the merger is

$$
A_{\text {initial }}=2 \times 4 \pi\left(2 m_{i}\right)^{2}=32 \pi m_{i}^{2} .
$$


The area of the resultant black hole is

$$
A_{\text {final }}=4 \pi\left(2 m_{f}\right)^{2}=16 \pi m_{f}^{2} .
$$

To maximise the energy dissipated in the merger the area of the final black hole must be as low as the Hawking area theorem allows, which is $A_{\text {final }}=A_{\text {initial }}$. So equating areas gives

$$
M_{f}=\sqrt{2} m_{i} .
$$

Thus the fraction of the initial mass lost in the merger is $1-2^{-1 / 2}=0.29$.

Problem 69 The total horizon area before the merger is

$$
A_{i}=2 \times 4 \pi\left(m_{K}\right)^{2}
$$

and the area of the final Schwarzschild black hole is

$$
A_{f}=4 \pi\left(2 m_{s}\right)^{2} .
$$

Equating the area before to the area after gives $m_{K}=m_{S}$. So half of the initial mass has been radiated away.

Problem 70 In the limit as $r \rightarrow \infty$ the first term inside the curly brackets reduces to $\omega^{2}$ and the other two terms tend to zero. So the Teukolsky equation simplifies to

$$
\frac{d^{2} u}{d r_{*}^{2}}=-\omega^{2} u
$$

which has equation (4.24) as its solution. In the limit as $r \rightarrow r_{+}, \Delta \rightarrow 0$ so in this case the Teukolsky equation reduces to

$$
\frac{d^{2} u}{d r_{*}^{2}}=\frac{\left[\left(r_{+}^{2}+a^{2}\right) \omega-a M\right]^{2} u}{\left(r_{+}^{2}+a^{2}\right)^{2}}=-\left(\omega-\omega_{+} M\right)^{2} u
$$

as $\left(r_{+}^{2}+a^{2}\right)=2 m r_{+}$and $\omega_{+}=a / 2 m r_{+}$. Thus in this limit the Teukolsky equation has equation (4.25) as its solution. Putting $a=0$ in the Teukolsky equation gives

$$
\frac{d^{2} u}{d r_{*}^{2}}+\omega^{2} u-\frac{l(l+1)(1-2 m / r) u}{r^{2}}-\frac{(1-2 m / r)^{2}}{r^{2}}-\frac{d}{d r_{*}}\left(\frac{(1-2 m / r)}{r}\right) u=0 .
$$

As

$$
\frac{d}{d r_{*}}=\frac{\Delta}{r^{2}+a^{2}} \frac{d}{d r}
$$

the last term becomes $(1-2 m / r)^{2} / r^{2}-2 m(1-2 m / r) / r^{3}$; so finally the Teukolsky equation reduces to

$$
\frac{d^{2} u}{d r_{*}^{2}}+\omega^{2} u-\frac{(1-2 m / r)[l(l+1)+2 m / r]}{r^{2}}=0
$$

which is the Regge-Wheeler equation. 
Problem 71 We are given that $W=u^{\prime} v-v^{\prime} u=$ constant for any two independent solutions $u$ and $v$ of a second order differential equation. A solution of equation (4.17) in the limit as $r_{*} \rightarrow-\infty$ is $u \sim e^{-i \omega r_{*}}$ (equation 4.18). Another solution is $v=u^{*}$. So differentiating $u$ and $v$ with respect to $r_{*}$ gives $u^{\prime}=-i \omega e^{-i \omega r_{*}}$ and $v^{\prime}=i \omega e^{i \omega r_{*}}$. Hence $W=-2 i \omega$. In the limit as $r_{*} \rightarrow+\infty$ the solutions to (4.17) takes the form $u \sim a_{\text {out }} e^{i \omega r_{*}}+a_{\text {in }} e^{-i \omega r_{*}}$ and $v=u * \sim a_{\text {out }} e^{-i \omega r_{*}}+a_{\text {in }} e^{i \omega r_{*}}$. Differentiating with respect to $r_{*}$ to get $u^{\prime}$ and $v^{\prime}$ we get a second expression for $W$ :

$$
W=2 i \omega\left(a_{\text {out }}^{2}-a_{\text {in }}^{2}\right) .
$$

Equating these two expressions gives $1+a_{\text {out }}^{2}=a_{\text {in }}^{2}$ the relation quoted in the text as equation (4.20).

In a similar way we can repeat this calculation for the limiting solutions of the Teukolsky equation to get the relation (4.26).

Problem 72 For a black hole radiating as a black body at temperature $T$ we can write $k T \sim h \nu=h c / \lambda$ where $\lambda$ is the wavelength at the peak of the blackbody spectrum. Now $\lambda$ will be of order oft he dimensions of the black hole (the only length scale in the problem), hence of order the Schwarzschild radius. So substituting $\lambda=2 G M / c^{2}$ into the above expression gives $T \sim h c^{3} / 2 k G M$.

Problem 73 To show that $U_{\omega^{\prime}}^{(+)}=\frac{1}{2\left(\pi\left|\omega^{\prime}\right|\right)^{1 / 2}} e^{-i\left|\omega^{\prime}\right| \eta} \xi^{i \omega^{\prime} / a}$ is a solution of the equation

$$
-\frac{1}{a^{2} \xi^{2}} \frac{\partial^{2} \Phi}{\partial \eta^{2}}+\frac{1}{\xi} \frac{\partial \Phi}{\partial \xi}+\frac{\partial^{2} \Phi}{\partial \xi^{2}}=0
$$

The factor $2\left(\pi\left|\omega^{\prime}\right|\right)^{-1 / 2}$ will cancel out so we can ignore it. Differentiating $U_{\omega}^{+}=$ $e^{-i\left|\omega^{\prime}\right| \eta} x i^{i \omega^{\prime} / a}$ twice with respect to $\eta$ gives

$$
\frac{\partial^{2} U}{\partial \eta^{2}}=i^{2} \omega^{\prime 2} e^{i \omega^{\prime} \eta} \xi^{i \omega^{\prime} / a}
$$

Differentiating $U_{\omega}^{(+)}$with respect to $\xi$ gives

$$
\frac{\partial U}{\partial \xi}=e^{-i \omega^{\prime} \eta}\left(i \omega^{\prime} / a\right) \xi^{-1+i \omega^{\prime} / a}
$$

and differentiating a second time gives

$$
\frac{\partial^{2} U}{\partial \xi^{2}}=e^{-i \omega^{\prime} \eta}\left(i \omega^{\prime} / a\right)\left(i \omega^{\prime} / a-1\right) \xi^{-2+i \omega^{\prime} / a} .
$$

Now substituting these three partial derivatives into the wave equation the quantity $e^{-i\left|\omega^{\prime}\right| \eta} \xi^{-2+i \omega^{\prime} / a}$ cancels leaving $-\frac{i^{2} \omega^{\prime 2}}{a^{2}}+\frac{i \omega^{\prime}}{a}+\frac{i^{2} \omega^{\prime 2}}{a^{2}}-\frac{i \omega^{\prime}}{a}=0$ so equation (4.34) is a solution of equation (4.33). 
Problem 74 From the definition of $r_{*}$ given in equation (4.16)

$$
\frac{r_{*}}{2 m}-1=\frac{r}{2 m}+\log \left(\frac{r}{2 m}-1\right)-1 .
$$

So near $r=2 m$

$$
\frac{r_{*}}{2 m}-1 \sim \log \left(\frac{r}{2 m}-1\right)
$$

and

$$
\frac{r}{2 m}-1 \sim 1-\frac{2 m}{r} \sim \kappa^{2} \xi^{2}
$$

where the second equality comes from equation (2.39). Combining (74) and (74) gives

$$
\kappa^{2} \xi^{2} \sim \exp \left(\frac{r_{*}}{2 m}-1\right)
$$

Now,

$$
U=-\exp \left(\frac{-u}{2 m}\right)
$$

and $u=t-r_{*}$. So

$$
U=-e^{r_{*} / 4 m} e^{-\kappa t} .
$$

Using (74) and $\kappa=1 / 4 m$ we get $U \propto \xi e^{-\kappa t}$.

Similarly the Kruskal coordinate $V=\exp (v / 4 m)$ near to $r=2 m$ becomes $V \propto \xi e^{\kappa t}$. The transformation from Rindler coordinates $(t, \xi)$ to Minkowski null coordinates $u=T-X$ and $v=T+X$ is given by $u=\xi e^{-\kappa \tau}$ and $v=\xi e^{\kappa \tau}$ using (2.41). Thus near $r=2 m$ the metric in Kruskal coordinates has approximately the Minkowski form in null coordinates.

Problem 75 The entropy of a black hole is given by $S=\frac{k c^{3} A_{h}}{4 \hbar G}$, where $A$ is the area of the horizon. For a spherical black hole the horizon area is $4 \pi\left(2 m_{p l}\right)^{2}$ in geometrical units. In physical units this becomes $4 \pi\left(2 G M_{p l} / c^{2}\right)^{2}$ and $M_{p l}=(\hbar c / G)^{1 / 2}$. So $A_{h}=$ $16 \pi \hbar G / c^{3}$ and hence $S=4 \pi k$.

Problem 76 Substituting $M=2 \times 10^{30} \mathrm{~kg}$ into the equation given in the text gives $t_{h}=\left[8.33 \times 10^{19} \times\left(2 \times 10^{30} / 10^{12}\right)^{3}\right] / 3.16 \times 10^{7}=2.2 \times 10^{67}$ years.

Problem 77 A black hole with a mass of $1.6 \times 10^{16} \mathrm{~kg}$ has a lifetime of $t_{h} \sim 2.5 \times$ $10^{9} \times(1.6)^{3}$ y or $1.02 \times 10^{10} \mathrm{y}$. Assume a typical gamma ray energy is $1 \mathrm{MeV}$. This corresponds to a temperature of $\sim 10^{10} \mathrm{~K}$. Using $T_{h} \sim 10^{23} / M$ K gives $M \sim 10^{13} \mathrm{~K}$ so such a black hole will have an age of order the age of the universe.

Problem 78 We have, $k T=\frac{\hbar c^{3}}{8 \pi G M}$. Setting $M=(G / \hbar c)^{-\frac{1}{2}}$, the Planck mass, gives $k T=\frac{1}{8 \pi}\left(\frac{\hbar c^{5}}{G}\right)^{\frac{1}{2}}$ so $T=7.78 \times 10^{7} \mathrm{~K}$. We also have $S=\frac{k c^{3}}{4 \hbar G} A_{h}$ and $A_{h}=1 \mathrm{~m}^{2}$ so $S=k\left(\frac{c^{3}}{4 \hbar G}\right)=1.3 \times 10^{46} \mathrm{JK}^{-1}$. 
Problem 79 The relationship between $M$ and $T$ is $M=\frac{\hbar c^{3}}{8 \pi G k T}$ (see section 4.6.1) so

$$
\frac{d\left(M c^{2}\right)}{d T}=-\frac{\hbar c^{5}}{8 \pi G k T^{2}}
$$

Expressing $T$ in terms of $M$ the result follows.

Problem 80 Let $x=M_{e q} / M_{i}$ and find the value of $x$ that maximises $(1-x) x^{4}$ by differentiating with respect to $x$ and setting to zero. This gives

$$
-x^{4}+4(1-x) x^{3}=0 .
$$

Thus $x=4 / 5$. Substituting this value of $x$ back in to the original expression gives $(1-4 / 5)(4 / 5)^{4}=0.82$.

Problem 81 To obtain the surface gravity of a Reissner-Nordstrom black hole we find the acceleration of a hovering observer by repeating the steps in section 2.8.1 for the Schwarzschild case, only this time with $g_{00}=g_{11}^{-1}=\left(1-2 m / r+q^{2} / r^{2}\right)$. This gives for the acceleration $a=\left(m / r^{2}-q^{2} / r^{3}\right)\left(1-2 m / r+q / r^{2}\right)^{-\frac{1}{2}}$. To get the acceleration measured by an observer at infinity we use equation (2.36) from section 2.8.2. with the Reissner-Nordstrom red shift factor replacing the Schwarzschild factor. This gives for the surface gravity $\kappa=\frac{\left(m r_{+}-q^{2}\right)}{r_{+}^{3}}$, where $r_{+}=m+\left(m^{2}-q^{2}\right)^{\frac{1}{2}}$. An extreme Reissner-Nordstrom black hole has $q=m$ and $r_{+}=m$ hence $\kappa=0$. And so $T=\hbar \kappa /(2 \pi c k)=0$.

Problem 82 The metric is

$$
d \tau^{2}=d t^{2}-d l^{2}-\left(b_{0}^{2}+l^{2}\right)\left(d \theta^{2}+\sin ^{2} \theta d \phi^{2}\right) .
$$

In the $\theta=\pi / 2$ plane at constant $t$ the spatial metric is

$$
d L^{2}=d l^{2}+\left(b_{0}^{2}+l^{2}\right) d \phi^{2} .
$$

The metric of Euclidean space is

$$
d L^{2}=d z^{2}+d r^{2}+r^{2} d \phi^{2} .
$$

On the surface $z=z(r)$ this becomes

$$
d L^{2}=\left[1+\left(\frac{d z}{d r}\right)^{2}\right] d r^{2}+r^{2} d \phi^{2} .
$$

Equations (14) and (15) are the same if

$$
\left(1+z^{\prime 2}\right) d r^{2}=d l^{2} \quad \text { and } \quad r^{2}=b_{0}^{2}+l^{2} .
$$


Thus

$$
1+z^{\prime 2}=\left(\frac{d r}{d l}\right)^{2}=\left(\frac{r}{l}\right)^{2}
$$

Integrating this equation gives

$$
z=b_{0} \cosh ^{-1}\left(\frac{r}{b_{0}}\right)
$$

Problem 83 Let the falling body have 4 -velocity components $u^{\mu}$. Since the metric is static we have, as usual, $u_{0}=E$. Now, a hovering (stationary) observer has 4 -velocity $u_{\mathrm{H}}^{0}=d t / d \tau=e^{-\Phi}$ so

$$
u_{\mu} u_{\mathrm{H}}^{\mu}=E e^{-\Phi}=\gamma=\left(1-v^{2}\right)^{-1 / 2}
$$

from which

$$
v=\left(1-\frac{e^{2 \Phi}}{E^{2}}\right)^{\frac{1}{2}} .
$$

The speed $v$ is that measured by the local stationary observer, hence $v=$ (proper distance) $/$ (proper time of the local observer $)=d l / e^{\Phi} d t$. The time to traverse the wormhole is obtained by integration.

Problem 84 Starting from the metric

$$
d \tau^{2}=d t^{2}-\frac{d r^{2}}{1-b_{0}^{2} / r^{2}}-\left(b_{0}^{2}+l^{2}\right)\left(d \theta^{2}+\sin ^{2} \theta d \phi^{2}\right)
$$

we set $\theta=\pi / 2$ and $d \tau^{2}=0$. The constants of the motion are $u_{0}=E_{p h}$ and $u_{3}=-L_{p h}$. Then $g_{\mu \nu} u^{\mu} u^{\nu}=0$ gives

$$
\left(\frac{d r}{d \lambda}\right)^{2}=E_{p h}^{2}\left[1-\frac{L_{p h}^{2}}{E_{p h}^{2} r^{2}}\right]\left(1-\frac{b_{0}^{2}}{r^{2}}\right) .
$$

For $L_{p h} / E_{p h}>b_{0}$ the first bracket on the right is zero for $r>b_{0}$. This is the point of closest approach: the radial coordinate of the light ray increases past this turning point and the light ray escapes to infinity. For $L_{p h} / E_{p h}<b_{0}$ the light ray enters the wormhole. The dividing value gives the impact parameter $L / E=b_{0}$. The capture cross section is then $\pi b_{0}^{2}$ because $r$ is an area coordinate.

Problem 85 The 4 -velocity of a static observer is $u^{\mu}=\left(e^{-\Phi}, 0,0,0\right)$ and the 4 acceleration is

$$
a^{\mu}=\frac{D u^{\mu}}{d \tau}=\frac{d u^{\mu}}{d \tau}+\Gamma_{\rho \sigma}^{\mu} u^{\rho} u^{\sigma} .
$$


We have $a^{\mu} u_{\mu}=a^{0} u_{0}=0$ so $a^{0}=0$. Also, $a^{2}=a^{3}=0$ by symmetry. Thus

$$
\begin{aligned}
a^{1} & =\Gamma_{00}^{1}\left(u^{0}\right)^{2} \\
& =\frac{1}{2} g^{11}\left(-\frac{\partial e^{2 \Phi}}{\partial r}\right)\left(u^{0}\right)^{2} \\
& =-\left(1-\frac{r}{r}\right) \Phi^{\prime} .
\end{aligned}
$$

The acceleration $a$ is now obtained from $a^{2}=a^{\mu} a_{\mu}$ as

$$
a=\left(1-\frac{b}{r}\right)^{\frac{1}{2}} \Phi^{\prime} .
$$

Problem 86 We have $u^{\mu} v_{\mu}=\gamma$ and $g^{\mu \nu} v_{\mu} v_{\nu}=1$ from which the result follows with some simple algebra.

Problem 87 If $\rho>0$ and $p=-\rho$ them $p+\rho=0$ which is consistent with the weak energy condition. In the frame of an observer moving at 4 -velocity $v^{\mu}$ relative to the fluid the energy density is

$$
T^{0^{\prime} 0^{\prime}}=\gamma^{2}\left(\rho+v^{2} p\right)=\gamma^{2}\left(1-v^{2}\right) \rho=\rho=T^{00} .
$$

Problem 88 As both stars are in circular orbits, from Newton's second law,

$$
\frac{\nu_{s}^{2}}{r_{s}}=\frac{G M_{d}}{r^{2}} .
$$

From the definition of the centre of mass of the system, $M_{s} r_{s}=M_{d} r_{d}$, therefore the distance $r$ between the two stars is

$$
r=r_{s}\left(1+\frac{M_{s}}{M_{d}}\right)
$$

The orbital period of the system is

$$
P=2 \pi r_{s} / \nu_{s}
$$

Combining (17) and (18) gives $r_{s}=\frac{G M_{d}}{\nu_{s}^{2}\left(1+M_{s} / M_{d}\right)^{2}}$ and substituting for $r_{s}$ from (19) gives $\frac{P \nu_{s}^{3}}{2 \pi G}=\frac{M_{d}}{\left(1+\left(M_{s} / M_{d}\right)^{2}\right)}$. But $\nu_{s}$ is the velocity in the plane of the orbit; this is related to the line of sight velocity by $V_{s}=\nu_{s} \sin \phi$, so finally

$$
\frac{P V_{s}^{3}}{2 \pi G}=\frac{M_{d} \sin ^{3} \phi}{\left(1+M_{s} / M_{d}\right)^{2}}
$$

Problem 89 From problem $15 t=2 \pi\left(r^{3} / m\right)^{\frac{1}{2}}$. Setting $r=6 m$, the radius of the last stable orbit, $t=2 \times 6^{3 / 2} \pi m$. Converting to physical units gives $t=2 \times 6^{3 / 2} \pi G M / c^{3}$ $=28.3$ minutes. 
This page intentionally left blank 


\section{References}

\section{Chapter 1}

Hartle J 2003 Gravity, An Introduction to Einstein's General Relativity (Addison Wesley)

Kenyon I R 1990 General Relativity (Oxford University Press)

Will C 1993 Theory and Experiment in Gravitational Physics (Cambridge University Press, Cambridge UK)

\section{Chapter 2}

Doeleman S S et al 2008 Nature 45578

Frolov V P and Novikov I D 1998 Black Hole Physics (Kluwer, Dordrecht)

Oppenheimer J R and Snyder H 1939 Phys Rev 5645

Taylor J H 1994 Rev Mod Phys 66711

Taylor E F and Wheeler J A 2000 Exploring Black Holes (Addison Wesley Longman) Thorne K S, Price R H and Macdonald D A 1986 Black Holes: The Membrane Paradigm (Yale University Press, New Haven)

\section{Chapter 3}

Bardeen J M et al 1972 Astrophys J 178347

Bardeen J M 1970 Nature 22664

Ciufolini I et al 1998 Science 2792100

Frolov V P and Novikov I D 1998 Black Hole Physics (Kluwer, Dordrecht/London) Lynden-Bell D 1978 Physica Scripta 17185

Begelman M and Rees M 1998 Gravity's Fatal Attraction (Scientific American Library)

Thorne K S 1974 Astrophys J 191507

Townsend P Black Holes, www.damtp.cam.ac.uk/lecturenotes/BH.ps (unpublished lecture notes) 


\section{Chapter 4}

Bekenstein J D 1975 Phys Rev D12 3077

Bekenstein J D 1980 Black Hole Thermodynamics Physics Today 24

Birrell N D and Davies P C W 1982 Quantum fields in Curved Spacetime (Cambridge University Press)

Chandrasekhar S 1983 The Mathematical Theory of Black Holes (Oxford University Press)

Custodio and Horvath 2003 Am J Phys 711237

Frolov V P and Novikov I D 1998 Black Hole Physics (Kluwer, Dordrecht/London)

Futterman J A H, Handler F A and Matzner R A, 1988 Scattering from Black Holes (Cambridge University Press, Cambridge UK)

Hawking S W 1974 Nature 24830

Hawking S W 1976 Phys Rev D13 191

Keifer C 1999 in Classical and Quantum Black Holes eds P Fré, V Gorini, G Magli and U Moschella (Institute of Physics, Bristol)

Takagi S 1986 Vacuum Noise and Stress Induced by Uniform Acceleration, Prog Theor Phys Supp 881

Townsend P K Black Holes, www.damtp.cam.ac.uk/lecturenotes/BH.ps (unpublished lecture notes)

Unruh 1976 Phys Rev D14 870

\section{Chapter5}

Bordag M, Mohideen U and Mostepanenko V M 2001 Physics Reports 353

Jensen B, McLaughlin J and Ottewill A 1988 Class. Quantum Grav. 5 L187-L189

Morris M S and Thorne K S 1988 Am J Phys 56395

Morris M S, Thorne K S and Yurtsever U 1988 Phys Rev Lett 6113

Thorne K S 1994 Black Holes and Time Warps: Einstein's Outrageous Legacy (Picador)

Visser M 1995 Lorentzian Wormholes (AIP) 


\section{Chapter 6}

Bardeen J M and Pettersen J A 1975 Astrophys J 105 L65

Barkana L H and Loeb A 2003 Nature 421341

Burgay M et al 2003 Nature 426531

Cottam J et al 2002 Nature 42051

Gebhardt K, Rich R M and Ho L 2002 Astrophys J L41 578

Genzel R et al 2003 Nature 425934

Ghez A et al 2008 Astrophys J 6891044

Begelman M and Rees M 1998 Gravity's Fatal Attraction (Scientific American Library)

Hartle J 2003 Gravity, An Introduction to Einstein's General Relativity (Addison Wesley)

Hawking S W and Ellis G F R 1973 The Scale Structure of Spacetime (Cambridge University Press)

Hjorth J et al 2003 Nature 423847

MacGibbon J and Carr B 1991 Astrophys J 371447

McLintock J E et al 2003 Astrophys J 593435

Melia F and Falcke H 2001 Ann Rev Astron Astrophys 39342

Narayan R, Garcia M and McClintock J E 2002 in Proc IX Marcel Grossmann Meeting eds V Gurzadyan, R T Jantzen and R Ruffini (World Scientific) p405

Orosz J A et al 2007 Nature 449872

Price R H 1972 Phys Rev D5 2410

Taylor J H 1994 Rev Mod Phys 66711

Van Der Marel R P et al 2002 Astron J 1243255

Zhang S N and Cui W 1998 Astrophys J 482 L155 
This page intentionally left blank 


\section{Bibliography}

The following is a selection of books on black hole physics at various levels.

Thorne K S 1994 Black holes and time warps (Papermac): gives a non-mathematical historical survey and includes the problems of time travel.

Luminet J-P 1987 Black holes (Cambridge University Press): a semi-popular exposition.

Taylor E F and Wheeler J A 2000 Exploring black holes (Addison Wesley Longman): an introduction for those with a basic knowledge of general relativity but without using tensor calculus.

Lightman A P et al 1974 Problem book in relativity and gravitation (Princeton): problems for advanced students (with outline answers).

Fre P et al ed 1999 Classical and quantum black holes (Institute of Physics): monograph on current developments in black holes and superstrings with an introductory first chapter.

Chandrasekhar S 1983 The Mathematical theory of black holes (Oxford): comprehensive mathematical treament of classical black hole spacetimes.

Frolov V P and Novikov I D 1998 Black hole physics (Dordrecht; London: Kluwer): detailed survey of black including quantum and astrophysical aspects.

Frank J, King A R and Raine D J 2002 Accretion power in astrophysics (Cambridge University Press): astrophysics of gas flows round compact objects. 
This page intentionally left blank 


\section{Index}

'down' modes, 117

'in' modes, 116

'no hair' property, 66

'out' modes, 117

'trans-Planckian' modes, 135

'up' modes, 117

4-acceleration, 11

4-momentum, 7, 9

4-velocity, 7

aberration, 49

accretion, 30, 95, 96, 101, 110, 111, 155, 157

active galaxies, 2, 157

affine connection, 5, 108

affine parameter, 11

angular momentum, 20, 23, 31, 67, 69, $72,85,110$

angular velocity, 72,74

apparent horizon, 58

area law, 112

area theorem, 111, 113

asymptotic flatness, 55

binary pulsar, 36, 153

binary stars, 154, 159

binding energy, 30, 85, 159

Birkoff's Theorem, 19

Birkoff's theorem, 36

black hole evaporation, 138

Boyer-Lindquist coordinates, 62

Carter integral, 68, 69

Casimir effect, 126

Casimir energy, 147

Cauchy horizon, 100
Chandrasekhar mass, 152

charged black hole, 103

chronology protection, 150

classical tests, 36

closed time-like lines, 102, 149

collapsing star, 50

conformal factor, 55

conformal transformation, 55

constant acceleration, 45

contravariant components, 8, 64

coordinate singularity, 46, 87, 97

cosmic censorship, 52, 100, 103

covariant components, 8

covariant derivative, 9

cross section, 79

cross-section, 34, 41

Cygnus X-1, 154, 160

Eddington-Finkelstein coordinates, 47, 50

effective potential, $28,71,78,116$

light, 33

Newtonian, 20

relativistic, 23, 25

Einstein-Rosen bridge, 53

embedding spacetime, 53, 143

energy, 9, 20, 22, 26, 30, 43, 67, 68, 110, 113

energy eigenstates, 128, 129

energy extraction, 114

energy momentum tensor, 12

energy-momentum tensor, 146

entropy, 3, 120, 122, 125, 135, 139, 140

equation of state, 153

equivalence principle, 2,3 
ergosphere, 80, 90, 107, 160

escape velocity, 1

event horizon, 2, 15, 19, 48, 51, 58, 63, 155

exotic matter, 147

extreme Kerr, 66, 83, 84, 87, 93, 97, 109, 111, 114, 139

field equations, 11

frame dragging, 72,88

free fall, 4, 21, 36, 67

frozen stars, 39

Galactic centre, 155

gamma-ray bursts, 153

geodesic, 21, 22

geodesic equation, 7

geometric mass, 18

geometric units, 62,65

geometrical optics, 132

geometrical units, 18

globular clusters, 158

gravitational collapse, 36, 52, 102, 151

gravitational lensing, 154

gravity waves, 154

Hawking, 107

Hawking radiation, 120, 125

Hawking temperature, 122, 125, 135, 139

horizon, 48, 98, 104

horizon area, 63, 110, 111, 136

hovering observer, 29, 75

impact parameter, 33, 41, 79

inertial frame, 4, 72

inertial observer, 127

infalling observer, 48

infinite redshift surface, 57

ingoing Kerr coordinates, 97

iron lines, 160

irreducible mass, 113

Israel's Theorem, 19 jets, 160

Kerr interior, 101

Kerr metric, 62, 64

Kerr-Newman metric, 103

Kerr-Schild coordinates, 99

Killing vector, 13

Killing's equation, 12

Kruskal coordinates, 50, 133

Lens-Thirring effect, 88, 90, 156

Lie derivative, 10

lifetime, 137

light ray, 11

lightlike interval, 6

local energy, 26, 76

Lorentz factor, 9

luminosity, 39, 136

maximal Kerr, 66

membrane paradigm, 58

metric

horizon, 44

Minkowski, 44

Minkowski metric, 6, 55

Minkowski spacetime, 55

naked singularity, 100, 114

negative energy, 90, 92, 126, 128, 132

negative frequency, 126

neutrinos, 153

neutron star, 152

neutron stars, 1

Newtonian

metric, 25

Newtonian gravity, 5

Newtonian metric, 56

Newtonian potential, 2, 5

null coordinates, 46

orbit, $25,35,66$

bound, 21,71

circular, 21, 27, 30, 31, 79, 81, 84, 88,90 
innermost stable, 30, 111

last stable, 84, 85, 93-95

light rays, 30

marginally bound, 25, 86

marginally stable, $28,82,83,85$

negative energy, 91,94

Newtonian, 20, 28

photon, 31, 77

polar, 87

radial, 31

relativistic, 21

unbound, 21, 71, 87

unstable, 28

orbits

bound, 28

parallel transport, 9

Penrose process, 93, 112, 160

Penrose-Carter diagram, 56, 98, 100

Penrose-Carter diagrams, 55

Planck energy, 136

Planck length, 136

Planck mass, 136

Planck spectrum, 131, 134, 137

Planck time, 136

Poisson's equation, 6

precession, 87, 90

primordial black holes, 159

proper distance, 17

proper time, 17

quantum gravity, 3

quantum vacuum, 126

quasi-normal modes, 118

radial infall, $26,36,40,46,48$

redshift, 2, 17, 38, 43, 81, 108

reference frame, 4

reflection coefficient, 117

Regge-Wheeler equation, 116, 134

Reissner-Nordstrom metric, 104, 139

relativistic energy, 23

relativistic wave equation, 115, 118, 127
Rindler coordinates, 44, 128

ring singularity, 99

Robinson's theorem, 66

Sagittarius A*, 156, 157

scalar product, 8

Schwarzschild

Karl, 15

metric, 15

radius, 18

Schwarzschild radius, 15

singularity, 51

naked, 51

slow rotation, 61,66

Smarr formula, 110, 113

spacelike interval, 6

spacetime

interval, 6

metric, 6

static, 19

stationary, 19

specific heat, 137

spherical harmonics, 115

spherical symmetry, 15

spontaneous emission, 119

star-burst galaxies, 157

static limit, 78, 79, 98, 107

summation convention, 5

supernova, 153

superradiance, 115, 119

surface gravity, 42, 44, 45, 107, 124, 133

test mass, 24

Teukolsky equation, 119, 134

thermodynamics, 3, 107

first law, 124

second law, 120, 124

third law, 97, 114, 125

theromdynamics

zeroth law, 124

tidal force, 40

time machines, 148

timelike interval, 6 
tortoise coordinate, 116

transmission coefficient, 117

trapped surface, 58

traversible wormholes, 143

uniform acceleration, 127, 128

vacuum energy, 147

weak energy condition, 146

white dwarfs, 1

white hole, 50, 51 wormhole, 55, 145

wormhole metric, 143

wormholes, 103, 141

x-ray binary, 154

x-ray polarisation, 160

x-rays, 160

ZAMOs, 75, 93, 107

Zerelli, 118

zero point energy, 126 